\title{
Who is seeking help for sleep? A clinical profile of patients in a sleep psychology clinic
}

\author{
${ }_{1}$ Annie Vallières ${ }^{1,2,3}$ Ph.D., Alric Pappathomas ${ }^{1}$ B.A., Taís Araújo ${ }^{1,5}$ Ph.D., Megan R. Crawford ${ }^{4}$ Ph.D., \\ and Séverine de Billy Garnier ${ }^{1,2,3}$ Ph.D. \\ ${ }^{2}$ École de psychologie, Pavillon Félix-Antoine-Savard, 2325 rue des Bibliothèques, Université Laval, \\ ${ }^{3}$ Québec, Québec, Canada, G1V 0A6. \\ ${ }^{4}$ Centre de recherche CERVO, 2525 de la Canardière, Québec, Québec, Canada, G1J 2G3. \\ ${ }^{5}$ Centre de recherche du CHU de Québec-Université Laval, Québec, Québec, Canada \\ School of Psychological Sciences and Health, University of Strathclyde, Glasgow, United Kingdom \\ HALEO Preventive Health Solutions Inc., Montréal, Québec, Canada, H3B 2E3
}

Corresponding author:

Annie Vallières, M.Ps., Ph.D.

2325 rue des Bibliothèques,

École de psychologie, Pavillon Félix-Antoine-Savard, local 1044

Université Laval, Québec, Québec, Canada, G1V 0A6

Phone: +1 418 656-2131 ext. 408258, e-mail: annie.vallieres@psy.ulaval.ca

ORCID: Annie Vallières: 0000-0002-9433-7880; Megan Crawford: 0000-0002-3167-1398 (the others authors do not have an ORCID number)

\section{Acknowledgments}

The authors wish to thank Claude Bomal (Vision MS) for his rigorous comments on the manuscript.

\section{Conflict of interest declaration and financial support:}

Fundings: This research did not receive any specific grant from funding agencies in the public, commercial, or not-for-profit sectors.

Conflict of interest: A. Vallières has received two grants from the Canadian Institutes of Health Research (CIHR: \#191771;\#110254) but they are not related to this paper. She is director of the Unité d'intervention pour les troubles du sommeil at the Service de consultation of the École de psychologie of Université Laval. Psychotherapy is delivered by graduate students in psychology under their program curses and following A. Vallières supervision. Patients pay some fees for receiving psychotherapy. A. Vallières developed clinical training video for JSCSleep clinic, and she is consultant for SHUSH since June 2019.

Alric Pappathomas, Taís Araújo, Megan Crawford, and Séverine de Billy Garnier: These authors declare that they have no conflict of interest. 
Running head: Clinical profile of patients in a sleep psychology clinic

Who is seeking help for sleep? A clinical profile of patients in a sleep psychology clinic Anonymous document for masked review process 
Clinical profile of patients in a sleep psychology clinic

\begin{abstract}
Background: The present study outlines a clinical profile of an ecologically valid population consulting for sleep difficulties at the Sleep Psychology Clinic of (masked for review).

Methods: Patients self-report to the sleep clinic. Following a phone screening interview, patients present to the clinic for a semi-structured clinical interview for sleep and psychopathology, which is conducted by psychologists and doctorate psychology students. A chart review of adult patients (56\% female, $\mathrm{M}_{\mathrm{age}}=43.6 \mathrm{yrs}$ ) was conducted (between 2015 and 2018) to record diagnosed sleep, psychiatric, and medical conditions.

Results: There was a high level of comorbidity with an average of 2.85 diagnoses (any diagnosis combined) per patient $(\mathrm{SD}=1.76)$, with $27 \%$ of the patients having at least four diagnoses. Reviewing specific types of disorders, $58.5 \%$ of patients presented with at least one comorbid psychiatric disorder, $27.5 \%$ with one medical comorbidity, or $39.5 \%$ with another sleep disorder alongside their primary sleep concern. Insomnia was the main sleep disorder (76\%). Anxiety (77.8\%) and depression (53.8\%) were the predominant psychiatric disorders, while fibromyalgia (10.9\%), hypertension $(10.9 \%)$, and head trauma $(9.1 \%)$ were the main medical conditions. Of patients with five diagnoses and more, $77.8 \%$ were taking on average 3.2 different types of medications. The number of diagnoses predicted the use of prescribed hypnotics and the use of any type of medications.

Conclusions: This clinical profile emphasizes the reality of multiple morbidities, which may have implications for clinical decisions. Future research is required to evaluate transdiagnostic approaches for the sleep disorder patient with multiple morbidities.
\end{abstract}

Key words: Insomnia; Sleep disorders; Psychology; Comorbidities; Transdiagnostic 
Clinical profile of patients in a sleep psychology clinic

Who is seeking help for sleep? A clinical profile of patients in a sleep psychology clinic

Sleep disturbances are known to be related to several psychiatric and medical conditions [1]. Sleep disturbances refer to sleep difficulties, poor sleep quality, insomnia, sleep apnea or other sleep disorders, including nightmares, somnambulism, or hypersomnia. There is a growing interest in the study of sleep disturbances with comorbidities, as reflected by the number of meta-analyses or reviews on the topic [2-5]. However, most of the evidence base surrounding non-pharmacological treatments for sleep disorders are on insomnia. Furthermore, the evidence stems from heavily regulated clinical trials that are restricted to patients with insomnia only or to patients with insomnia comorbid to one disorder only. For instance, cognitive behavioral therapy for insomnia (CBT-I) was evaluated in a sample of people with insomnia and breast cancer [6], with insomnia and PTSD [7], or with insomnia and mild sleep disorder breathing [8]. Moreover, patients taking hypnotic medications or a combination of hypnotics are often excluded from clinical trials or included only if they agree to maintain stable doses and intake during the trial, see for example the criteria use in CBT-I studies [9-12].

In the anxiety and depression fields, there are reviews highlighting that clinical trial samples are not representative of the patients seen in clinics [13-15]. These studies demonstrated that 70 to $82 \%$ of patients seeking treatment for their anxiety or depression in their clinic would have been excluded from research studies. We seek to study this phenomenon in the sleep disorders field. To study the representativity of the trial samples in the sleep field, we need to describe the clinical profile of patients who are seeking help for sleep in primary care and psychological settings. Then, a further step would be to compare these profiles with those of patients in clinical trials described in the scientific literature. This should help to represent the patient's reality and should facilitate in providing evidence-based care in the context of multiple morbidity. Some studies have already reported patients clinical profile from primary care settings [16-19] or the prevalence of a specific sleep disorder in a sleep clinic population [20]. However to our knowledge, there are no available clinical profiles of patients from a psychological sleep clinic. Translating clinical observations into research might inform future research 
Clinical profile of patients in a sleep psychology clinic agenda and strengthen the link between research and clinic. It could also favor the development of personalized management of sleep disorders, as recommended [21]. In an attempt to link scientific knowledge and clinical comprehension, the present study outlines a clinical profile of patients attending to our sleep psychology clinic.

\section{Method}

The psychological sleep clinic

The psychological sleep clinic at (masked for review) values a personalized treatment approach. The advertised service offer is intended for anyone with sleep disturbances or with the daytime consequences related to these difficulties. The clinic opened in February 2015 and was advertised by the first author in medical clinics, the two public sleep apnea clinics, and on the radio in the (masked for review) area. The sleep clinic provides an evidence-based treatment for insomnia and other sleep disorders to the general population. The clinical team is composed of three psychologists, possessing 30 years of accumulative experience in sleep research and the clinic setting. They offer individual therapy and clinical supervision to graduate psychology students. Patients paid $\$ 50$ per hour for a trainee and $\$ 80$ for a psychologist. Fees are covered by private health insurance and tax deductible. Patients

Patients are self-referred to the sleep psychology clinic. The sleep clinic brochures were distributed to a general practitioner or tertiary provider (e.g., pulmonologist in public hospital) who could suggest to their patients to consult at our clinic. E-mails detailing the sleep clinic service were also sent to the (masked for review) community. The inclusion criterion for the chart review was that of being an adult and there were no exclusion criteria. From February 2015 to May 2018, 470 patients contacted the clinic. Of these, 181 did not wish to conduct the initial screening for a variety of reasons, such as no longer being interested in psychological help, no longer wanting to pay for the service, or being unreacheable after several attempts of contact. The remaining 289 accepted the initial screening and the phone interview was conducted. Of these, 82 patients were no longer interested in receiving 
Clinical profile of patients in a sleep psychology clinic psychological help, following the phone interview. 207 patients, including 200 adults reported here and 7 children (not reported here), signed the consent form, were assessed, and treated.

Procedures for patient intake in the psychological sleep clinic

Prior to intake 15-minute telephone interviews are conducted to determine patient complaint.

Once a primary sleep complaint is established, individuals undergo an in-person clinical evaluation. Psychologists and graduate psychology students perform the evaluation under the supervision of clinical psychologists (masked for review). Students possess at least two years of clinical experience before being trained in our clinic. The in-person clinical evaluation consists of the Structured Insomnia Interview (SII [22]) to screen for insomnia and other potential sleep disorders. Subsequently, the MiniInternational Neuropsychiatric Interview (M.I.N.I [23]), a semi-structured clinical interview, is used to screen for psychiatric conditions. The interviews also capture sociocultural aspects (e.g., work, family background) and sleep disorder history with more opened questions. The procedure follows the evaluation guidelines [24] and encourages patients to actively participate in meetings with health professionals, as suggested by Rocque and colleagues [25]. Therapists assigned a score for each patient's insomnia severity based on two questions of the Insomnia Severity Index (ISI) [26] included in the SII [22]. These questions were answered on a Likert scale ranging from " 0 " to " 4 ". If the patient scored of "3" or "4" its insomnia was considered severe, insomnia was considered moderate with a score of "2", and mild with a score of "1". Sleep disorder diagnoses such as sleep related breathing disorders or hypersomnia are made by a pneumologist before referring the patient and confirmed through correspondence with the referring specialist. Only patients referred by a pneumologist were screened for these sleep disorders. Psychiatric conditions severity was not ranked as the DSM-5 [27] does not provide severity specification criteria for all of them. Current medical conditions and medications are self-reported and not confirmed by the GP. For this reason, their severity was not ranked. Considering that data were collected from 2015 to 2018, the International Statistical Classification of Diseases and related health problems, tenth revision (ICD-10 [28]) was used for the 
Clinical profile of patients in a sleep psychology clinic classification of the medical conditions. Previous medications intake was self-reported but was not included in this study to prevent retrospective recall bias. For the purpose of this study, sleep disorders are considered a distinct category from psychiatric and medical conditions, although we note that some of them fall into psychiatric conditions (e.g., insomnia) or medical conditions (e.g., restless legs syndrome). Each interview of the in-person evaluation lasted for a maximum of 120 minutes. Patients completed several self-reported questionnaires and sleep diaries (not reported here).

Procedures for chart review

The design used for this study is a chart review. Undergraduate research assistant extracted anonymous clinical data, under the supervision of the second author (masked for review). A participant number was attributed to each patient by the second author (masked for review) to maintain the data anonymity. The research was approved by the (masked for review) ethical committee (\#2017-319). Informed consent was obtained for all individuals in the study prior to the in-person evaluation.

Frequency and descriptive analyses were performed on the following variables: 1) number of sleep disorders, 2) number of psychiatric conditions, 3) number of medical conditions, and 4) current medication use (hypnotic or non-hypnotic medication and prescribed or non-prescribed medication). Medications with number of diagnoses were also cross-tabulated. Two logistic regressions were performed to evaluate the relationship between the number of diagnoses and the likelihood of taking medication or not. The first one was conducted to predict the use of prescribed hypnotics (dependant variable) and the second one, the use of any type of medications (dependant variable) with the number of diagnoses as the independent variable.

\section{Results}

The treated patients mean age was 43.6 years $(\mathrm{SD}=13.62 ; 18-84$ years old $)$ and $56 \%$ were women. Patients endorsed an average of 2.85 diagnoses $(\mathrm{SD}=1.76$; median $=3)$, which could include sleep disorders, psychiatric, and/or a medical condition. Of the sample, $16 \%$ experienced only one diagnosis, while $27 \%$ of the patients experienced four or more diagnoses. For sleep diagnoses, $92.5 \%$ 
Clinical profile of patients in a sleep psychology clinic of patients presented with at least one sleep disorder, while $7.5 \%$ of patients had sleep disturbance symptoms without meeting the criteria for a sleep disorder (they may suffer from an anxiety disorder and have sleep difficulties but not meet full criteria for a sleep disorder). In addition, $39.5 \%$ of patients presented with two or more sleep disorders. For psychiatric conditions, $58.5 \%$ of all the patients presented with at least one psychiatric condition. About $1 / 4$ of patients $(26.5 \%)$ with at least one sleep disorder also had two or more psychiatric conditions. Figure 1 shows the patients distribution sorted by the total number of sleep disorders and illustrates the overlap with psychiatric and medical conditions.

$$
\overline{\text { Insert Figure } 1 \mathrm{~A} \text { and } \mathrm{B}}
$$

Insomnia was the most prevalent sleep disorder with $76.0 \%$ of patients meeting DSM-5 [27] criteria for insomnia disorder, followed by sleep apnea (26.5\%), fatigue with no identifiable sleep disorder (17.5\%), circadian rhythm sleep disorders (7\%), and hypersomnia (5\%). The therapist who conducted the SII [21] rated $27.9 \%$ of the insomnia cases as being severe, $55.8 \%$ moderate, and $16.3 \%$ mild. The main psychiatric conditions were anxiety (77.8\%) and depression (53.8\%). Among anxiety diagnoses, $52.7 \%$ were generalised anxiety disorder and $18.7 \%$ social anxiety. Of the entire sample $27.5 \%$ self-reported a medical condition. In total, 35 different medical conditions were self-reported and fall under many different axes of the ICD-10 [28]. The main axes were endocrine, nutritional and metabolic diseases (axis IV), diseases of the nervous system (VI), diseases of the circulatory system (IX), and diseases of the musculoskeletal system and connective tissue (XIII). The main medical conditions reported were fibromyalgia (10.9\%), hypertension $(10.9 \%)$, and head trauma $(9.1 \%)$. Moreover, $16 \%$ of patients $(\mathrm{n}=32)$ presented with at least one disorder of each type (sleep, psychiatric, and medical). All of these 32 patients had one anxiety disorder and 59.4\% suffered additionally from depression. Furthermore, $91.7 \%$ of the insomnia diagnosis for these patients were severe or moderate and $8.3 \%$ were mild. 
Clinical profile of patients in a sleep psychology clinic

Table 1 shows descriptive statistics for medication intake in relation to number of diagnoses.

When considering one diagnosis, $31.3 \%$ of patients were taking at least one medication of any of the four categories with an average of 1.7 medication, and $90 \%$ of these patients took at least one prescribed hypnotic. The percentage of patients taking medications increased with the number of diagnoses. When considering five diagnoses or more, $77.8 \%$ of patients were taking on average 3.2 medications, and $86 \%$ of these patients took at least one prescribed hypnotic. Zopiclone and trazodone were the most frequently used prescribed hypnotics. The logistic regressions showed that the number of diagnoses predicted both the use of prescribed hypnotics, $\beta=.30, p=.001, O R=1.35$ (95\% CI: 1.12 , $1.63)$ and the use of any type of medications, $\beta=.44, p<.001, O R=1.56(95 \% \mathrm{CI}: 1.26,1.93)$.

\section{Insert Table 1}

\section{Discussion}

The clinical profile presented here demonstrates the reality of multiple morbidities encountered in patients at our sleep psychology clinic, with more than a quarter of the sample reporting at least four separates diagnoses. Among them, two out of five patients presented with at least two sleep disorders. These sleep disorders are mostly a combination of persistent insomnia, sleep apnea, circadian rhythm sleep disorder, and hypersomnia. Insomnia severity was mainly moderate and severe. They come along with the most frequent combination of psychiatric conditions that are generalized or social anxiety disorders as well as depression. Furthermore, the number of diagnoses predicts the use of prescribed hypnotics and the use of any type of medications.

The evaluation procedure applied clearly highlighted the co-existence of sleep disorders and psychiatric conditions in a majority of patients. The results raised several issues. First, it identifies more complex clinical cases than are typically included in research samples (see for example [9-11]) or reported in primary care case reports [16-19]. For example, Zailinawati et al. [19] found that, among patients with chronic insomnia, about $48 \%$ had anxiety symptoms and $37 \%$ had depression. We found 
Clinical profile of patients in a sleep psychology clinic higher comorbidity rates than expected, however this may also be explained by our screening methods. To be consistent with the active patient participation approach recommended for medical consultations [25], two semi-structured interviews and open-ended questions were used. This may have increased the likelihood of patients reporting symptoms and might explain why the comorbidity rates were so much higher. Second, there were fewer medical conditions in our psychological setting than in a primary care setting, such as the one reported by Davidson et al. [16] who found $83 \%$ of medical comorbidities. There are several explanations for this discrepancy. It might reflect the fact that in our clinic, medical conditions were self-reported, while in a medical setting they can be extracted from the medical file. The discrepancy might also reflect the patients' decision-making process in seeking help, as presented by Cheung and coll [29]. It has been shown that patients with insomnia may be more comfortable to seek help from a medical professional first [30,31], which may explain the higher rate of medical conditions observed in the primary care reports. However, in presence of several psychiatric comorbidities, patients' decision-making process could evolve towards ending up seeking help from a psychologist, thus explaining the higher rate of psychiatric conditions and complex cases observed in our psychological setting. The third issue our data highlights is that social anxiety disorder is frequently related to sleep disorders and insomnia. This result converges with previous results $[32,33]$ and supports that research is needed to further understand the relationship between sleep and social anxiety [4]. Furthermore, results on sleep disorders and social anxiety suggest that the diagnostic procedures should not be limited to insomnia and generalized anxiety.

The observation of multiple morbidities and the use of several medications has implications for treatment plans and informs future research agendas. First, the multiple sleep comorbidities indicates that we need more evidence-based behavioral and psychological treatments for sleep disorders outside of just insomnia to improve the non-pharmacological aid that can be provided to sleep disordered patients. There are a few studies that have embarked on this mission [34-37]. Another important issue is that multiple morbidities supports the idea of a transdiagnostic approach for sleep disorders, similar 
Clinical profile of patients in a sleep psychology clinic to that used for depression and anxiety $[38,39]$. Some scientific writings suggest that sleep disorders, anxiety, and depression are interconnected under common processes [40,41] as shown by the fact that CBT-I might have a moderate effect on comorbid disorder [42,43]. Already, some attempts have identified anxious arousal as a transdiagnostic variable between anxiety and insomnia [44], as well as anxiety sensitivity between anxiety, depression, and insomnia [45]. To properly develop transdiagnostic treatment, sleep researchers need to collaborate with non-sleep researchers in order to make waves in both areas. Research to evalutate transdiagnostic approaches within different sleep disorders is also an exciting avenue. An advantage of a transdiagnostic approach over an approach that favours one treatment per disorder is that it reduces the overall duration of treatment. It can also prevent that only one of the necessary treatments is applied, which would leave the patient with the residual symptoms of the other disorders. Thus, an effective transdiagnostic approach would follow the most recent guideline for the treatment of insomnia, which is the treatment of each disorder [46]. Finally, our findings beg the question as to whether our clinical samples are represented by published research samples. Most clinical trials on CBT-I have evaluated efficiency when insomnia is comorbid with a maximum of one other condition, which represents only $1 / 3$ of our clinical sample. Moreover, medication use is often restricted or excluded in these studies, further reducing the representativeness for our clinical patients. This statement about the lack of representativity of the clinical sample in research needs to be validated. A systematic review investigating the samples in clinical trials used to evaluate the efficacy of CBT-I is currently underway by our research group.

In the context of multiple medications that come with the number of diagnoses, psychological interventions, such as CBT-I, have to be adapted to the medication intake. The treatment adaptation can take several forms. When considering CBT-I, one has to take into consideration that each medication has a different effect on sleep duration, sleep stages, and daytime performance. For instance, trazodone and venlafaxine are commonly used for depression but trazodone induces much more sleepiness than venlafaxine, which is known for its stimulating effect. Therefore, two patients taking different 
Clinical profile of patients in a sleep psychology clinic medication combinations (zopiclone and trazodone versus zopiclone and venlafaxine) and are beginning CBT-I might need a different adaptation of the treatment. The patient taking zopiclone and trazodone is likely to feel sleepier during the day and before going to bed than the patient taking zopiclone and venlafaxine. The former may need another intervention to counter sleepiness during the day (e.g., power nap, light exercise, or light therapy) while the latter may need to modify his or her sleep schedule (e.g., going to bed later), taking into account the stimulating effect of venlafaxine. Research on CBT-I in the context of multiple comorbidities and medications is urgently needed.

The present study possesses strengths and limitations. The evaluation procedure we chose to apply is both a strength and a limitation. On the one hand, it provides an ecologically valid sample of patients attending a sleep psychology clinic. On the other hand, there was little experimental control, for example, medical conditions that psychologists are not permitted to evaluate (according to the laws of our region), were self-reported and for this reason could not reflect the true numbers. Sleep disorders such as sleep apnea or hypersomnia can also be underestimated for the same reason which means that those who were not referred by a pneumologist were not screened for these sleep disorders. The nature of the clinic may also affect the generalizability of the clinical profile identified. The fact that the clinic involved psychological trainees might have attracted a part of the population that cannot afford private psychological services. However, it is possible that many patients were not deterred by being treated by a trainee since it was the only specialized service available in the region when it opened. Finally, the methodology used in the present study involves patient observation. Thus, proportions obtained do not represent prevalence rates in the general population.

By providing a clinical profile of an ecologically valid population experiencing sleep difficulties, this study fills the gap between research and clinic. It highlights clinical issues (multiple morbidities, medicated conditions, sleep disorders other than insomnia) that must be addressed together in order to offer appropriate evidence-based treatment. The evaluation procedure used in this study can be easily replicated by non-sleep psychologists: It could thus be used in other psychological contexts to 
Clinical profile of patients in a sleep psychology clinic identify, for example, patients consulting for psychiatric conditions but experiencing sleep disorders. Our semi-structured interviews can be complemented by available questionnaires screening for multiple sleep disorders [47]. More clinical profiles drawn from different psychological sleep clinics should be published. Together, these efforts will ensure that treatments developed in research are generalizable to and suitable for the patients seen in sleep clinics.

Ethical approval:

All procedures performed in studies involving human participants were in accordance with the ethical standards of the institutional and/or national research committee and with the 1964 Helsinki declaration and its later amendments or comparable ethical standards. 
Clinical profile of patients in a sleep psychology clinic

Table 1. Descriptive statistics for medication intake in relation to number of diagnoses per patient

\begin{tabular}{|c|c|c|c|c|c|c|c|c|}
\hline \multirow[b]{2}{*}{ No. } & & \multicolumn{2}{|c|}{ Hypnotic $^{\mathrm{a}}$} & \multicolumn{2}{|c|}{ Non-hypnotic $^{\mathrm{a}}$} & \multicolumn{3}{|c|}{ Any medication ${ }^{b}$} \\
\hline & & & Non- & & Non- & & & \\
\hline $\mathrm{DX}$ & & Prescribed & prescribed & Prescribed & prescribed & & & \\
\hline per & $\mathrm{N}$ & $\%(n)$ & $\%(n)$ & $\%(n)$ & $\%(n)$ & $\%(n)$ & $\mathrm{M}(\mathrm{SD})$ & $\mathrm{Me}$ \\
\hline \multicolumn{9}{|c|}{ patient } \\
\hline 0 & 8 & $12.5(1)$ & $0.0(0)$ & $0.0(0)$ & $0.0(0)$ & $12.5(1)$ & $1.0(\mathrm{na})$ & 1.0 \\
\hline 1 & 32 & $28.1(9)$ & $3.1(1)$ & $3.1(1)$ & $0.0(0)$ & $31.3(10)$ & $1.7(1.3)$ & 1.0 \\
\hline 2 & 57 & $50.9(29)$ & $14.0(8)$ & $1.8(1)$ & $0.0(0)$ & $56.1(32)$ & $1.7(1.1)$ & 1.0 \\
\hline 3 & 49 & $63.3(31)$ & $8.2(4)$ & $16.3(8)$ & $10.2(5)$ & $67.3(33)$ & $2.5(2.5)$ & 2.0 \\
\hline 4 & 27 & $63.0(17)$ & $11.1(3)$ & $18.5(5)$ & $7.4(2)$ & $66.7(18)$ & $2.5(1.1)$ & 2.0 \\
\hline$\geq 5$ & 27 & $66.7(18)$ & $29.6(8)$ & $22.2(6)$ & $3.7(1)$ & $77.8(21)$ & $3.2(2.9)$ & 2.0 \\
\hline
\end{tabular}

\footnotetext{
${ }^{a}$ Medication was sorted into four categories (hypnotic or non-hypnotic medication and prescribed or nonprescribed medication);

${ }^{b}$ Any medication represents data relative to medication use whatever the categories of medication. The $n$ for any medication represents the number of patients taking at least one medication of the four categories. Note. No. $=$ number; $\mathrm{DX}=$ Diagnoses (medical, psychiatric and sleep); $\mathrm{N}=$ Total number of patients with either $0,1,2,3,4$, or $\geq 5$ diagnoses; $(n)=$ number of patients using medication with either $0,1,2$, 3,4 , or $\geq 5$ diagnoses; $\%=$ Percentage $\left(\mathrm{n} / \mathrm{N}^{*} 100\right)$ of patients using medication with either $0,1,2,3,4$, or $\geq 5$ diagnoses; $M$ (SD) = Mean (Standard Deviation) of medications taken per patient using medication; $\mathrm{Me}=$ Median of medications taken per patient using medication; na $=$ not applicable.
} 
Clinical profile of patients in a sleep psychology clinic

Figure caption

Figure $1 \mathrm{~A}$ and $1 \mathrm{~B}$

Patients sorted by the number of sleep disorders diagnoses they received.

Note. No. $=$ Number; PSY DX $=$ Psychiatric condition; SLEEP DX = sleep diagnosis; MED DX $=$ Medical condition 
Clinical profile of patients in a sleep psychology clinic

\section{References}

[1] Ohayon MM, Caulet M, Lemoine P. Comorbidity of mental and insomnia disorders in the general population. Compr Psychiatry. 1998; 39: 185-197.

[2] Baglioni C, Battagliese G, Feige B, et al. Insomnia as a predictor of depression: a meta-analytic evaluation of longitudinal epidemiological studies. J Affect Disord. 2011; 135: 10-9.

[3] Belleville G, Cousineau H, Levrier K, St-Pierre-Delorme ME. Meta-analytic review of the impact of cognitive-behavior therapy for insomnia concomitant anxiety. Clin Psychol Rev.2011;31: 638-52.

[4] Cox RCOlatunji BO. A systematic review of sleep disturbance in anxiety and related disorders. $J$ Anxiety Disord. 2016; 37: 104-29.

[5] Taylor DJPruiksma KE. Cognitive and behavioural therapy for insomnia (CBT-I) in psychiatric populations: a systematic review. Int Rev Psychiatry. 2014; 26: 205-13.

[6] Savard J, Simard S, Ivers H, Morin CM. Randomized study on the efficacy of cognitive-behavioral therapy for insomnia secondary to breast cancer, part I: Sleep and psychological effects. $J$ Clin Oncol. 2005; 23: 6083-6096.

[7] Talbot LS, Maguen S, Metzler TJ, et al. Cognitive behavioral therapy for insomnia in posttraumatic stress disorder: a randomized controlled trial. Sleep. 2014; 37: 327-41.

[8] Guilleminault C, Davis K, Huynh NT. Prospective randomized study of patients with insomnia and mild sleep disordered breathing. Sleep. 2008; 31: 1527-33.

[9] Edinger JD, Wohlgemuth WK, Radtke RA, Coffman CJ, Carney CE. Dose-response effects of cognitive-behavioral insomnia therapy: a randomized clinical trial. Sleep. 2007; 30: 203-12.

[10] Morin CM, Vallieres A, Guay B, et al. Cognitive behavioral therapy, singly and combined with medication, for persistent insomnia: a randomized controlled trial. JAMA. 2009; 301: 2005-15.

[11] Germain A, Richardson R, Stocker R, et al. Treatment for insomnia in combat-exposed OEF/OIF/ OND military veterans: preliminary randomized controlled trial. Behav Res Ther. 2014; 61: 78-88.

[12]Lichstein KL, Wilson NM, Johnson CT. Psychological treatment of secondary insomnia. Psychol Aging. 2000; 15: 232-40.

[13] Hoertel N, Le Strat Y, Blanco C, Lavaud P, Dubertret C. Generalizability of clinical trial results for generalized anxiety disorder to community samples. Depress Anxiety. 2012; 29: 614-20.

[14] Hoertel N, de Maricourt P, Katz J, et al. Are participants in pharmacological and psychotherapy treatment trials for social anxiety disorder representative of patients in real-life settings? $J$ Clin Psychopharmacol. 2014; 34: 697-703.

[15]Zimmerman M, Mattia JI, Posternak MA. Are subjects in pharmacological treatment trials of depression representative of patients in routine clinical practice? Am J Psychiatry. 2002;159:469-73.

[16] Davidson JR, Dawson S, Krsmanovic A. Effectiveness of Group Cognitive Behavioral Therapy for Insomnia (CBT-I) in a Primary Care Setting. Behav Sleep Med. 2019; 17: 191-201.

[17] Jaisoorya TS, Dahale AB, Sunil KG, et al. Insomnia in primary care-a study from India. Sleep Health. 2018; 4: 63-67.

[18] Shochat T, Umphress J, Israel AG, Ancoli-Israel S. Insomnia in primary care patients. Sleep. 1999; 22 Suppl 2: S359-65.

[19]Zailinawati AH, Mazza D, Teng CL. Prevalence of insomnia and its impact on daily function amongst Malaysian primary care patients. Asia Pac Fam Med. 2012; 11: 9.

[20] Curtis BJ, Ashbrook LH, Young T, et al. Extreme morning chronotypes are often familial and not exceedingly rare: the estimated prevalence of advanced sleep phase, familial advanced sleep phase, and advanced sleep-wake phase disorder in a sleep clinic population. Sleep. 2019. 10.1093/sleep/zsz148.

[21]Zee PC, Badr MS, Kushida C, et al. Strategic opportunities in sleep and circadian research: report of the Joint Task Force of the Sleep Research Society and American Academy of Sleep Medicine. Sleep. 2014; 37: 219-27.

[22] Morin CM. Insomnia: Psychological assessment and management.1993, New York: Guilford Press. 
Clinical profile of patients in a sleep psychology clinic

[23] Sheehan DV, Lecrubier Y, Sheehan KH, et al. The Mini-International Neuropsychiatric Interview (M.I.N.I.): the development and validation of a structured diagnostic psychiatric interview for DSM-IV and ICD-10. J Clin Psychiatry. 1998; 59 Suppl 20: 22-33;quiz 34-57.

[24] Ong JC, Arnedt JT, Gehrman PR. Insomnia Diagnosis, Assessment, and Evaluation., in Principles and Practice of Sleep Medicine 2017. p. 785-93.e4. .

[25] Rocque R, Levesque A, Leanza L. Patient participation in medical consultations: the experience of patients from various ethnolinguistic backgrounds. Patient Exp J. 2019; 6: 19-30.

[26] Bastien CH, Vallieres A, Morin CM. Validation of the Insomnia Severity Index as an outcome measure for insomnia research. Sleep Med. 2001; 2: 297-307.

[27] American Psychiatric Association, ed. Diagnostic and statistical manual of mental disorders. ${ }^{5 \mathrm{e}}$ ed. 2013, American Psychiatric Association: Washington, DC.

[28] World Health Organization. ICD-10 : international statistical classification of diseases and related health problems : tenth revision, 2nd ed. 2004: World Health Organization.

[29] Cheung JMY, Bartlett DJ, Armour CL, Laba TL, Saini B. To Drug or Not to Drug: A Qualitative Study of Patients' Decision-Making Processes for Managing Insomnia. Behav Sleep Med. 2018; 16: 1-26.

[30]Cheung JM, Bartlett DJ, Armour CL, Glozier N, Saini B. Insomnia patients' help-seeking experiences. Behav Sleep Med. 2014; 12: 106-22.

[31] Morin CM, LeBlanc M, Daley M, Gregoire JP, Merette C. Epidemiology of insomnia: prevalence, self-help treatments, consultations, and determinants of help-seeking behaviors. Sleep Med. 2006; 7: 123-30.

[32] Kushnir J, Marom S, Mazar M, Sadeh A, Hermesh H. The link between social anxiety disorder, treatment outcome, and sleep difficulties among patients receiving cognitive behavioral group therapy. Sleep Med. 2014; 15: 515-21.

[33] Stein MB, Kroft CD, Walker JR. Sleep impairment in patients with social phobia. Psychiatry Res. 1993; 49: 251-6.

[34] Jarnefelt H, Lagerstedt R, Kajaste S, et al. Cognitive behavioral therapy for shift workers with chronic insomnia. Sleep Med. 2012; 13: 1238-46.

[35] Neikrug AB, Crawford MR, Ong JC. Behavioral Sleep Medicine Services for Hypersomnia Disorders: A Survey Study. Behav Sleep Med. 2017; 15: 158-171.

[36]Lee KA, Gay CL, Alsten CR. Home-based behavioral sleep training for shift workers: a pilot study. Behav Sleep Med. 2014; 12: 455-68.

[37] Vallières A, Roy M, Bastille-Denis E, Claveau S, Simon T. Exploring a behavioral therapy for Insomnia in shift workers. J Sleep Disor Ther. 2015; 4.

[38] Arnfred SM, Aharoni R, Hvenegaard M, et al. Transdiagnostic group CBT vs. standard group CBT for depression, social anxiety disorder and agoraphobia/panic disorder: Study protocol for a pragmatic, multicenter non-inferiority randomized controlled trial. BMC Psychiatry. 2017; 17: 37.

[39] Owens VAM, Hadjistavropoulos HD, Schneider LH, et al. Transdiagnostic, internet-delivered cognitive behavior therapy for depression and anxiety: Exploring impact on health anxiety. Internet Interv. 2019; 15: 60-66.

[40] Dolsen MR, Asarnow LD, Harvey AG. Insomnia as a transdiagnostic process in psychiatric disorders. Curr Psychiatry Rep. 2014; 16: 471.

[41] Harvey AG. A transdiagnostic approach to treating sleep disturbance in psychiatric disorders. Cogn Behav Ther. 2009; 38 Suppl 1: 35-42.

[42] Geiger-Brown JM, Rogers VE, Liu W, et al. Cognitive behavioral therapy in persons with comorbid insomnia: A meta-analysis. Sleep Med Rev. 2015; 23: 54-67.

[43] Wu JQ, Appleman ER, Salazar RD, Ong JC. Cognitive Behavioral Therapy for Insomnia Comorbid With Psychiatric and Medical Conditions: A Meta-analysis. JAMA Intern Med. 2015; 175: 1461-72. 
Clinical profile of patients in a sleep psychology clinic

[44] McGowan SK, Espejo EP, Balliett N, Werdowatz EA. The Effects of Transdiagnostic Group CBT for Anxiety on Insomnia Symptoms. Cogn Behav Ther. 2016; 45: 163-75.

[45]Fairholme CP, Carl JR, Farchione TJ, Schonwetter SW. Transdiagnostic processes in emotional disorders and insomnia: results from a sample of adult outpatients with anxiety and mood disorders. Behav Res Ther. 2012; 50: 522-8.

[46] Riemann D, Baglioni C, Bassetti C, et al. European guideline for the diagnosis and treatment of insomnia. J Sleep Res. 2017; 26: 675-700.

[47]Klingman KJ, Jungquist CR, Perlis ML. Questionnaires that screen for multiple sleep disorders. Sleep Med Rev. 2017; 32: 37-44. 


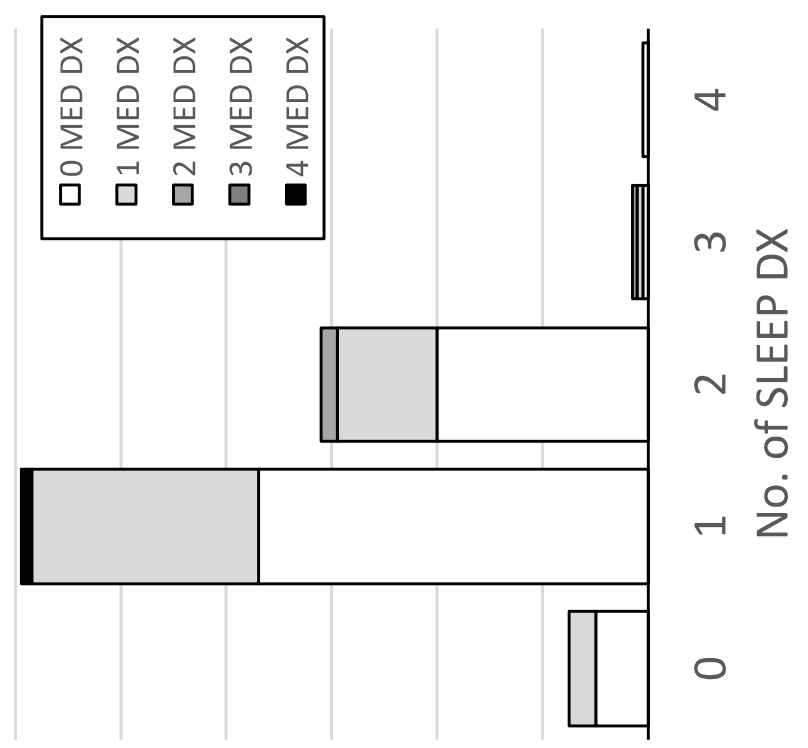

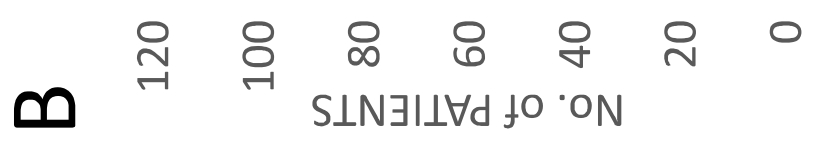

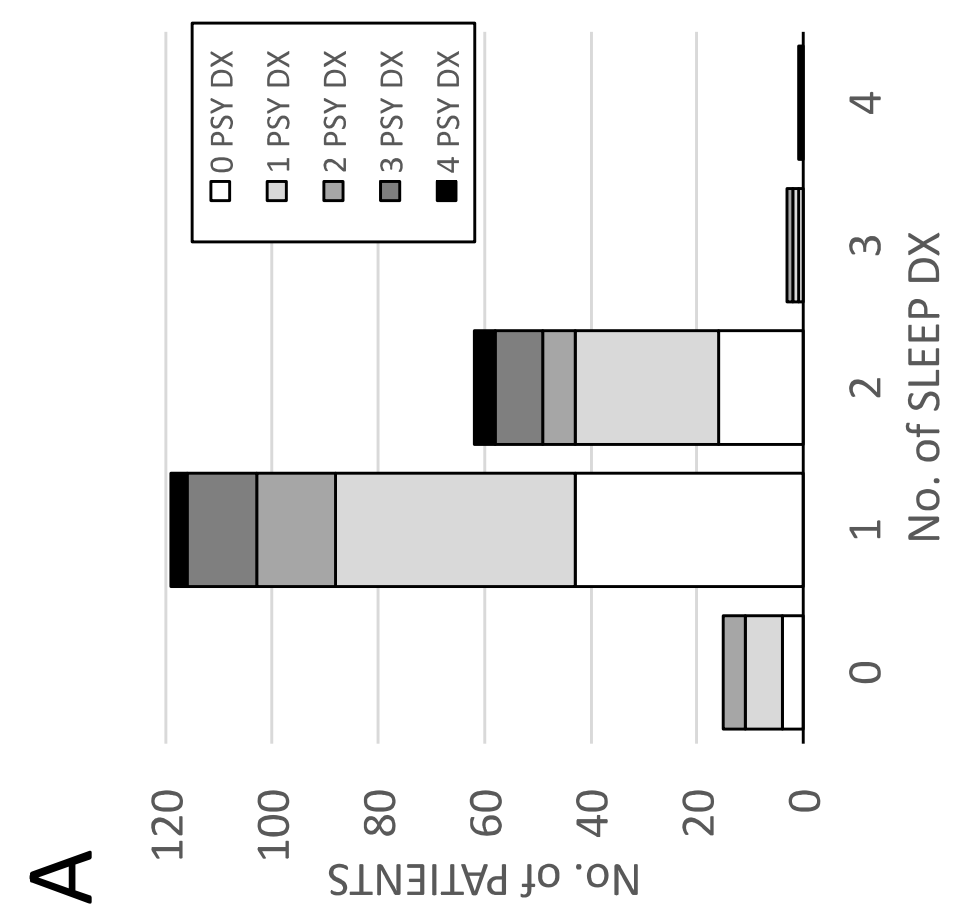

